\title{
FATORES QUE AFETAM O ENCANTAMENTO DO CLIENTE DO TURISMO DE LAZER A PARTIR DA EXPERIÊNCIA EM PARQUES TEMÁTICOS
}

FACTORS AFFECTING CUSTOMER DELIGHT OF LEISURE'S TOURISM FROM THE THEME PARK'S EXPERIENCE

\section{FACTORES QUE AFECTAN AL ENCANTO DE CLIENTES DE TURISMO DE OCIO CON LA EXPERIENCIA DE LOS PARQUES TEMÁTICOS}

Fabíola Fernandes Silva

Doutoranda em Turismo, Mestra em Turismo e Bacharela em Turismo pela Universidade Federal do Rio Grande do Norte - UFRN

fabiola_fsd@yahoo.com.br

\section{Sérgio Marques Júnior}

Docente no Programa de Pós-Graduação em Turismo da Universidade Federal do

Rio Grande do Norte Doutor em agronomia pela Universidade Estadual Paulista Júlio de Mesquita Mestre em Agronomia pela Universidade de São Paulo sergio@ct.ufrn.br

Data de Submissão: 16/05/2016

Data de Aprovação: 21/10/2016

RESUMO: Com a crescente demanda pela instalação de empreendimentos no ramo do entretenimento, observa-se o aumento da competitividade neste setor, gerando a necessidade de diferenciação em meio aos demais, visando à fidelização de clientes, o que pode ser idealizado a partir da perspectiva do "Encantamento do Cliente". Nesse contexto, o presente estudo teve o objetivo de investigar as inter-relações entre os fatores antecedentes capazes de influenciar o encantamento do cliente do turismo de lazer. Para tanto, a investigação apresentou caráter exploratório-descritivo com enfoque analítico-quantitativo. A pesquisa foi do tipo Survey, com os dados sendo coletados a partir de 
um público composto por 176 adolescentes que viajaram em excursão para o Walt Disney World, localizado em Orlando, Flórida, em junho de 2014, por uma agência de viagem localizada na cidade de Natal/RN. O parque escolhido para objeto da pesquisa foi o Magic Kingdom. Para análise dos dados, foi utilizada a análise fatorial exploratória (AFE) com posterior análise de regressão linear múltipla. Pelas inter-relações encontradas, observou-se que as dimensões "Coerência com Objetivo", "Interesse pelo Objetivo" e "Inesperado", consideradas como antecedentes do Encantamento, têm impactos significativos na formação do Encantamento do Cliente. As constatações permitiram ampliar a compreensão sobre o comportamento do consumidor no turismo de lazer.

PALAVRAS-CHAVE: Turismo de Lazer e Entretenimento. Encantamento do Cliente. Parques Temáticos.

ABSTRACT: Due to the increased demand for projects in the entertainment business, there is increased competition in this sector, generating the need for differentiation among others, aimed at customer loyalty, from the perspective of "Customer Delight." In this context, this study aimed to investigate the interrelationships among factors that can influence the delight of leisure tourism customer. Therefore, this study presented descriptive exploratory-character with quantitative analytical approach. The Survey collected data from an audience of 176 adolescents who traveled in package tours to Walt Disney World, located in Orlando, Florida, in June 2014, by a travel agency located in the city of Natal/RN. The park chosen for the object of the survey was the Magic Kingdom. For data analysis was used exploratory factor technique and multiple linear regression analysis. It was noted that the dimensions: "Appetitive Goal Congruence", "Goal Interest" and "Unexpectedness", considered of Customer Delight background, have a significant impact on the Customer Delight formation. Findings advance the understanding of consumer behavior in leisure tourism.

KEYWORDS: Leisure Tourism and Entertainment. Customer Delight. Theme Parks.

RESUMEN: Con la creciente demanda de proyectos en el negocio del entretenimiento, hay una mayor competencia en este sector, lo que genera la necesidad de diferenciación entre otros, dirigidos a la lealtad del cliente. En este sentido, viene el término "Encantamiento del cliente." En este contexto, el presente estudio tiene como objetivo investigar, a través del análisis de regresión lineal múltiple, las interrelaciones entre los factores que pueden influir en el Encantamiento del cliente. Por lo tanto, la investigación presentada carácter exploratorio-descriptivo con enfoque analítico cuantitativo. La investigación fue del tipo Survey (Encuesta). Los datos se obtuvieron de una audiencia de 176 adolescentes que viajaban en paquetes turísticos a Walt Disney World, ubicado en Orlando, Florida, en junio de 2014, en una agencia de viajes en la ciudad de Natal/RN. El parque elegido para el objeto de la investigación fue el Magic Kingdom. Para el análisis de los datos, se utilizó la análisis factorial y análisis de regresión lineal múltiple. Las relaciones encontradas, se observó que las dimensiones "Compatibilidad con Propósito", "de Interés en el Ojetivo" y "inesperada", considerado como el fondo encantamiento, tienen un impacto significativo en la formación encantamiento cliente. Los hallazgos avanzar en la comprensión del comportamiento de los consumidores en el turismo de ocio.

PALABRAS CLAVE: Turismo de Ocio y Entretenimiento. Encantamiento del Cliente. Parques Tematicos.

INTRODUÇÃO

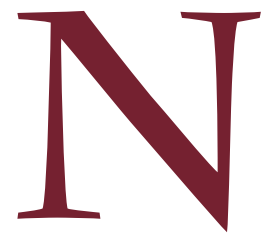

o ambiente competitivo em que se inserem os parques temáticos, cresce o empenho do empresariado na busca de oferecer aos consumidores experiências únicas e prazerosas como forma de diferenciação e, com isso, obter a fidelização de clientes. Elementos signficativos 
para atração de demanda no ramo de entretenimento, os parques temáticos são empreendimentos que utilizam temas diferenciados na ambientação física de suas atrações e têm como objetivo mercadológico o estímulo da atividade turística (Ansarah, 1999, p. 96). A visitação de parques temáticos é uma experiência na qual as emoções desempenham um papel importante na qualidade da experiência dos turistas (Ma, Gao, Scott, \& Ding,, 2013).

Estudos, como o proposto por Keiningham e Varva (2001), consideraram que pequenas diferenças nos níveis de satisfação têm pouca probabilidade de gerar lealdade. Nesta perspectiva, a satisfação apenas não era suficiente e o objetivo das empresas deveria ir um pouco mais adiante e oferecer algo além das expectativas dos consumidores (Almeida, 2003). Diante disto, surge o termo "Encantamento do Cliente" [Customer Delight], com características diferentes do termo "Satisfação". Enquanto a satisfação do cliente é geralmente baseada em atender as expectativas, encantamento do cliente requer que os clientes recebam uma surpresa positiva que está além de suas expectativas (Berman, 2005).

Neste sentido, o objetivo deste estudo foi investigar as inter-relações entre os fatores capazes de influenciar o Encantamento do Cliente. Para tanto, como objeto de investigação, o presente estudo adotou um parque temático como o contexto para análise. Trata-se do Magic Kingdom, parque temático do complexo turístico Walt Disney World, localizado em Orlando, Flórida, considerado um modelo eficiente de empresa que supera as expectativas dos seus clientes (Conellan, 2010). Com mais de 20 milhões de visitantes por ano, o Magic Kingdom é o parque temático mais visitado do mundo (TEA/AECOM, 2015), constituindo-se, portanto de um dos mais importantes atrativos turísticos mundiais.

A relevância deste estudo, do ponto de vista acadêmico, está no fato de contribuir com a construção de teorias que estudem os antecedentes do Encantamento do Cliente, diante a escassez de matérias que estudem o tema, tendo em vista que compreender os antecendentes das emoções pode ajudar os gestores a prever respostas emocionais dos consumidores decorrentes da experiência projetada (Ma et al., 2013). Entende-se também relevante, do ponto de vista acadêmico, o uso de adolescentes como foco de investigação, pois o mercado mundial deste segmento de mercado tem um importante 
impacto sobre a economia mundial, sendo esperado que, em 2020, cerca de 2,5 bilhões de pessoas, ou um terço da população do mundo, sejam menores de 18 anos (Gentina, Butori, Rose, \& Bakir, 2014). Entende-se que o estudo é relevante ainda, do ponto de vista gerencial, pelo fato de fornecer informações estratégicas que poderão auxiliar os gestores de turismo preocupados em criar uma experiência que incentive o surgimento do encantamento. Encantar os clientes pode proporcionar uma vantagem competitiva e resultar no aumento de vendas e lucro para a empresa, além da retenção de clientes.

Para oferecer subsídios teóricos a essa discussão, inicialmente são discutidos aspectos da gestão de empreendimentos turísticos de lazer e parques temáticos, seguidos da discussão sobre o Encantamento do Cliente. Na sequência, são apresentadas as características metodológicas do estudo e a análise dos resultados obtidos por meio da pesquisa de campo, encerrando com as conclusões do estudo e recomendações para futuras pesquisas.

\section{GESTÃO DE EMPREENDIMENTOS TURÍSTICOS DE LAZER E PARQUES TEMÁTICOS}

Em 2015, o turismo mundial apresentou um aumento significativo no número de chegadas de turistas internacionais $(4,4 \%)$ atingindo cerca de 1.184 milhões de chegadas. Em comparação com o ano anterior, em 2015 houve aumento de mais 50 milhões de turistas internacionais em todo o mundo (OMT, 2016). Gastos em viagens de lazer (receptivo e doméstico) geraram 76,6\% do PIB direto do setor de Viagens \& Turismo em 2015, em comparação com 23,4\% para gastos de viagens de negócios (WTTC, 2016).

No turismo de lazer, é possível identificar a existência de diversos empreendimentos específicos. Entende-se por empreendimentos turísticos, instalações e serviços indispensáveis ao desenvolvimento da atividade turística (Lohmann \& Panosso Netto, 2008; Barreto, 1991). Entre as várias categorias de empreendimentos turísticos, destaca-se a de entretenimento, que pode ser definida como "o conjunto das atividades relacionadas à qualidade de vida, e que se refere aos locais destinados a propiciar divertimento, dotados de equipamentos e serviços indispensáveis à atividade turística" (Ansarah, 1999, p.94). 
Os parques temáticos são empreendimentos do ramo do entretenimento que promovem divertimento e lazer e atraem uma rede de serviços de apoio e equipamentos de infraestrutura (Ansarah, 1999).

A definição que a maioria dos analistas adotam é a de parques temáticos são de lazer onde estão distintas atrações criadas a partir de um tema concreto como inspiração ou pano de fundo, presente em todos os componentes do parque, sejam eles equipamentos, design, arquitetura ou gastronomia (Gastal \& Castrogiovanni, 2003, p. 124).

A tendência é que esses empreendimentos venham a fomentar a economia local, através do surgimento de outras atividades comerciais complementares, tanto em seu entorno, como em seu interior (Santil, 2001, p.91). Além disso, podem aumentar a renda deste núcleo através do aumento do tempo de permanência dos visitantes, bem como, aumentar o gasto médio do turista. Esta estratégia é adotada, por exemplo, pelos Estados Unidos (Valente, 2006).

Com o aumento pela busca por empreendimentos que proporcionem lazer e entretenimento, entre eles os parques temáticos, é perceptível a existência da competição no setor. Nesta situação, há a necessidade de serem adotadas ações que promovam a diferenciação em meio aos demais, uma busca por uma vantagem competitiva (Chagas, 2010). É diante deste cenário que se potencializa a busca pela fidelização de clientes, indicador que mede a intenção dos consumidores voltarem a fazer compras à organização e a sua vontade em estabelecer atividades de parceria com a organização (Kotler, Bowen \& Makens, 1999). A fidelidade do cliente é uma questão estratégica para o sucesso de uma empresa. Retenção de clientes é mais desejável e menos caro do que atrair novos (Füller \& Matzler, 2008).

A atividade turística envolve pessoas, empresas, organizações e lugares, que se combinam de alguma forma para proporcionar alguma experiência de viagem (Gohr \& Santos, 2010). Entre a decisão de compra do turista e a realização desta compra, existem uma série de fatores pouco controlados, incluindo os relacionados estritamente com o serviço e sua prestação (Ramos \& Dias, 2010). Os serviços diferem fundamentalmente das mercadorias por sua intangibilidade. $\mathrm{O}$ atendimento nos serviços é um encontro entre pessoas, 
portanto, deste encontro pode nascer ou manifestar alguma emoção (Silva, 2004). As emoções podem ser utilizadas como uma forma de fidelização do cliente (Manosso, Gândara, Souza, \& Bógea, 2012).

As emoções contribuem para a formação da satisfação. Clientes satisfeitos podem apresentar diferentes níveis de fidelidade ou intenção de recompra (MA et al., 2013). "Há uma maior probabilidade dos turistas satisfeitos voltarem ao mesmo destino, como também estão mais dispostos a partilhar as suas experiências positivas de viagem com os seus amigos e parentes" (Santoro, 2014 , p.28). Visto que as empresas com clientes fiéis apresentam uma vantagem competitiva frente aos seus concorrentes e que o encantamento do cliente influencia a fidelidade do cliente, então, encantar os clientes representa uma estratégia competitiva para as empresas.

Características do Encantamento do Cliente são abordadas no próximo tópico.

\section{ENCANTAMENTO DO CLIENTE}

O termo "encantamento" aparece na literatura do comportamento do consumidor, por volta do inicio dos anos 90, como sendo um termo para designar um grupo de consumidores que estivesse vivenciando altos níveis de satisfação e de prazer com a experiência de consumo (Almeida \& Nique, 2007). Encantamento do Cliente é uma emoção relacionada ao consumo hedonista (MA et al., 2013) podendo ser entendido como uma avaliação pós-consumo (Kumar, 1996). A literatura da emoção sugere que encantamento é uma emoção positiva (Kwong \& Yau, 2002). Pode ser definida como a reação que os clientes têm quando experimentam um produto ou serviço que não só o satisfaz, mas fornece um nível imprevisto de valor ou satisfação (Crotts \& Magnini, 2011).

Compreender a capacidade de explicação do componente afetivo nas avaliações pós-consumo esbarra na comparação entre Satisfação e Encantamento (Almeida \& Nique, 2007). Encantamento do Cliente é um conceito mais recente que a Satisfação do Cliente (Schümmer, 2007). Encantamento é um constructo relacionado, mas separado de Satisfação, uma vez que se baseia em coisas diferentes (Berman, 2005). Satisfação e 
ISSN: 1983-7151

Encantamento do Cliente são efetivamente avaliações distintas à experiência de consumo (Almeida, 2003, p.39).

Encantamento e Satisfação também diferem em termos de seus antecedentes e do processo pelo qual eles são formados (Masroujeh, 2009). Satisfação é essencialmente uma avaliação cognitiva, enquanto que Encantamento é uma reação emocional (Robinson, 2012). Enquanto a Satisfação do Cliente é geralmente baseada em atender as expectativas, Encantamento do Cliente requer que os clientes recebam uma surpresa positiva que está além de suas expectativas (Berman, 2005).

Outra diferença é que os efeitos do Encantamento nas intenções de recompra vão além dos efeitos da Satisfação e há também uma maior probabilidade de os consumidores encantados utilizem do mecanismo de "boca a boca positivo" com relação àqueles que estão meramente satisfeitos. $O$ sentimento de encantamento não pode ser confundido com mera satisfação, ainda que o consumidor encantado deva primeiramente estar satisfeito (Almeida \& Nique, 2007). Portanto, Encantamento implica em uma emoção mais forte e um estado psicológico diferente do que Satisfação (Ariffin \& Yahaya, 2013, p. 34).

Encantamento do Cliente pode ser uma estratégia para aumento da competitividade organizacional (Torres, Fu, \& Letho, 2014), pois além de conduzir para a fidelidade, clientes encantados fornecem feedback de suas experiências positivas para os outros (Berman, 2005).

Estudos na área de Marketing baseiam suas considerações em abordagens de categorias e dimensões que classificam uma emoção em termos de valência positiva ou negativa e excitação alta ou baixa. A Teoria da Avaliação Cognitiva (Cognitive Appraisal Theory - CAT), por outro lado, prevê um mecanismo para a obtenção de uma emoção, considerando que os indivíduos avaliam suas experiências comparando com as suas motivações e usando dimensões de avaliação definidas (Ma et al, 2013). A teoria da Avaliação Cognitiva sugere que as emoções são originadas a partir de uma avaliação subjetiva ou avaliação de uma situação ou evento (Bee \& Neubaum, 2014) e as situações podem ser avaliadas ao longo de várias dimensões, sendo a combinação de um subconjunto dessas dimensões que cria uma emoção específica (Lerner \& Keltner, 2000). 
De acordo com a CAT, dois indivíduos expostos a um mesmo evento podem experimentar diferentes respostas emocionais (Bee \& Neubaum, 2014), pois não é o evento especifico que produz a emoção, mas a avaliação psicológica do evento que obtém a emoção discreta (Bee \& Neubaum, 2014). Emoção discreta refere-se a uma reação emocional que consiste em qualquer combinação das dimensões de avaliação, que podem abranger uma gama de rótulos emocionais identificados (Johnson \& Stewart, 2005).

Devido a superioridade desta teoria em oferecer explicações para os antecedentes e consequentes de emoções, há recomendações para aplicar esta teoria em estudos no turismo, no qual se verifica pouca discussão sobre os antecedentes das emoções e de como a experiência de consumo influencia a natureza de uma resposta emocional. A maioria dos estudos nesta área são focados nos efeitos das emoções sobre comportamentos pré e pós-consumo. Porém, compreender os antecendentes das emoções é teoricamente importante, uma vez que pode ajudar os gestores a prever respostas emocionais dos consumidores decorrentes da experiência projetada (Ma et al., 2013). Diferentes emoções podem ser originadas pela mesma situação quando turistas divergem em suas avaliações em suas dimensões de avaliação.

Dimensões de avaliação das emoções servem para diferenciar reações emocionais discretas que podem ocorrer em resposta à percepção de informações pessoais relevantes (Johnson \& Stewart, 2005). Estudos, como os propostos por Roseman (1991), Ellsworth (1985), Frijda (1987), Scherer (1988) e Frijda et al. (1988), identificaram numerosas dimensões de avaliação das emoções (Ma, 2013). Diferentes termos são usados para descrever as dimensões de avaliação incluídos nas várias teorias de avaliação, mas são baseados em conceitos semelhantes (Ma, 2013).

Ma et al. (2013) adotou um modelo de relacionamento, baseado na Teoria de Avaliação Cognitiva para estudar os antecedentes do Encantamento do Cliente. Esta abordagem postula que as emoções, e encantamento em particular, são provocadas pelas dimensões "Coerência com o objetivo", "Importância do objetivo", "Interesse pelo objetivo" e "Inesperado", que irão exercer influência sobre a avaliação de um indivíduo de uma situação, e assim provocar o 
surgimento de emoção positiva, resultando na emoção mais específica de encantamento.

De acordo com Ma et al. (2013), a dimensão "Coerência com o Objetivo" indica a valência (positiva ou negativa) de uma resposta emocional ao determinar se uma experiência em particular está relacionada com uma meta. Metas são benefícios abstratos que os turistas buscam ao consumir produtos ou serviços, cujas características ajudam a satisfazer suas necessidades (Hosany, 2012).

A dimensão "Importância do Objetivo" é a avaliação da relevância da experiência das metas ou necessidades do turista. A avaliação desta dimensão afeta a intensidade da emoção provocada, porque está associada com a conveniência do estado que se pretende obter ou a gravidade das consequências do fracasso. "Interesse pelo objetivo" é uma resposta geral para situações percebidas subjetivamente como importante e motiva altos níveis de atenção, portanto, aumentando as chances de provocar uma emoção positiva, como per exemplo a diversão.

A dimensão da "Inesperado" permite avaliar o grau em que uma experiência afasta as expectativas do turista. É interpretada como a rapidez ou "inesperado" de uma experiência e associado com o encantamento e surpresa.

Baseando-se no referencial apresentado, foi proposto o presente estudo cujos procedimentos metodológicos são apresentados e discutidos a seguir:

\section{METODOLOGIA}

A investigação proposta neste estudo apresentou caráter descritivo exploratório no que concerne aos seus objetivos, com uma abordagem analítica-quantitativa. Segundo a extensão do campo de estudo, a pesquisa foi do tipo Survey, ou seja, uma investigação com o objetivo de levantar dados ou informações sobre características, ações ou opiniões de determinado grupo de pessoas, por meio de um instrumento de pesquisa, normalmente um questionário.

A investigação foi desenvolvida com adolescentes, na faixa etária de 13 a 19 anos, que viajaram em excursões para os parques do Walt Disney World em 
Orlando, Flórida, no período de junho de 2014 por uma agência de turismo localizada na cidade de Natal/RN. O parque deste complexo escolhido para ser objeto da pesquisa foi o Magic Kingdom, atrativo visitado por uma média de 20.492.000 pessoas por ano (TEA/AECOM, 2015).

Pela impossibilidade de se conhecer com exatidão o tamanho da população assim como se obter uma listagem dos elementos da população ou unidades amostrais, o método de pesquisa utilizado foi o não probabilístico. Na constituição do instrumento de pesquisa, para garantir que os índices de validação das dimensões (fatores) a serem obtidas na investigação, fossem adequados, foi necessário coletar-se entre 10 a 15 observações por variável observável (Marôco, 2010). Baseando-se neste critério, em função no número de questões existentes no instrumento de pesquisa (questionário), seria necessária uma amostra de 150 entrevistados. Entretanto, a presente pesquisa coletou um número de 176 entrevistados, número acima do adequado para que seja possível atingir os objetivos de estudo.

Para fins de ajustamento dos dados, foram retirados os entrevistados que informaram faixa etária superior a 19 anos (05 questionários), uma vez que o foco da pesquisa são os adolescentes. Foi realizado um pré-teste, em maio de 2014, com oito adolescentes para avaliar a qualidade geral do questionário, tanto no que diz respeito à clareza da redação, sequência lógica, organização e a facilidade do entendimento das questões, assim como uma estimativa do tempo médio que seria gasto para preenchimento. Para a execução deste procedimento (pré-teste) foi utilizada a técnica de Grupo Focal como método de investigação.

O questionário final foi dividido em seis partes. Cinco partes relativas às seguintes dimensões entendidas como capazes de influenciar o encantamento do cliente, baseados em Ma et al. (2013): Coerência com o Objetivo, Importância do Objetivo, Interesse pelo Objetivo, Inesperado e Encantamento. A última parte do questionário continha questões do perfil do entrevistado.

A escala de avaliação utilizada foi baseada no modelo Likert, de onze pontos de opção de escolha de resposta, no qual o entrevistado era convidado a apresentar seu nível de concordância com uma determinada frase. As respostas 
variavam das opções 0,0 (zero), quando o entrevistado discordava plenamente da afirmação proposta até 10,0 (dez) quando o entrevistado concordava plenamente com a afirmação proposta. O questionário foi aplicado pós-visita ao parque para examinar as respostas emocionais dos visitantes. As coletas foram realizadas em junho de 2014 por guias responsáveis pela excursão, treinados previamente com a aplicação do instrumento da pesquisa.

As variáveis e dimensões utilizadas no estudo são apresentadas no quadro 01.

Quadro 01 - Variáveis observáveis e dimensões utilizadas no estudo

\begin{tabular}{|c|c|c|}
\hline Variável & Descrição da Variável & Dimensão \\
\hline COE1 & O Magic Kingdom conseguiu satisfazer todas as minhas expectativas. & \multirow{3}{*}{$\begin{array}{l}\text { Coerência com } \\
\text { o Objetivo }\end{array}$} \\
\hline COE2 & Eu atingi meus objetivos de lazer na visita ao Magic Kingdom. & \\
\hline COE3 & Eu tinha necessidade de me divertir que foi atingida com a visita ao parque. & \\
\hline IMP1 & Foi muito importante ter visitado este parque. & \multirow{3}{*}{$\begin{array}{l}\text { Importância } \\
\text { do Objetivo }\end{array}$} \\
\hline IMP2 & Visitar este parque significou muito para mim. & \\
\hline IMP3 & Considero a visita ao parque fundamental para este momento da minha vida. & \\
\hline INT1 & Eu tinha muita curiosidade sobre como seria o Magic Kingdom. & \multirow{3}{*}{$\begin{array}{l}\text { Interesse pelo } \\
\text { Objetivo }\end{array}$} \\
\hline INT2 & O parque sempre me chamou muita atenção. & \\
\hline INT3 & Eu tinha muito interesse em conhecer o Magic Kingdom. & \\
\hline INE1 & Eu não esperava encontrar no Magic Kingdom o que eu encontrei. & \multirow{3}{*}{ Inesperado } \\
\hline INE2 & Encontrei atrativos no Magic Kingdom que eu não esperava. & \\
\hline INE3 & Encontrei muitas situações inesperadas no Magic Kingdom. & \\
\hline ENC1 & Achei o Magic Kingdom incrível. & \multirow{3}{*}{ Encantamento } \\
\hline ENC2 & $\begin{array}{c}\text { Eu nunca vi um ambiente tão mágico quanto o encontrado no Magic } \\
\text { Kingdom. }\end{array}$ & \\
\hline ENC3 & Eu não teria palavras para descrever como foi boa a visita ao Magic Kingdom & \\
\hline
\end{tabular}

Fonte: Dados da pesquisa (2014).

O modelo de análise proposto neste estudo foi baseado no "Modelo dos Antecedentes do Encantamento do Cliente", proposto por Ma et al. (2013), apresentado na figura 01 : 
Figura 01 - Modelo dos antecedentes do Encantamento do Cliente proposto no estudo.

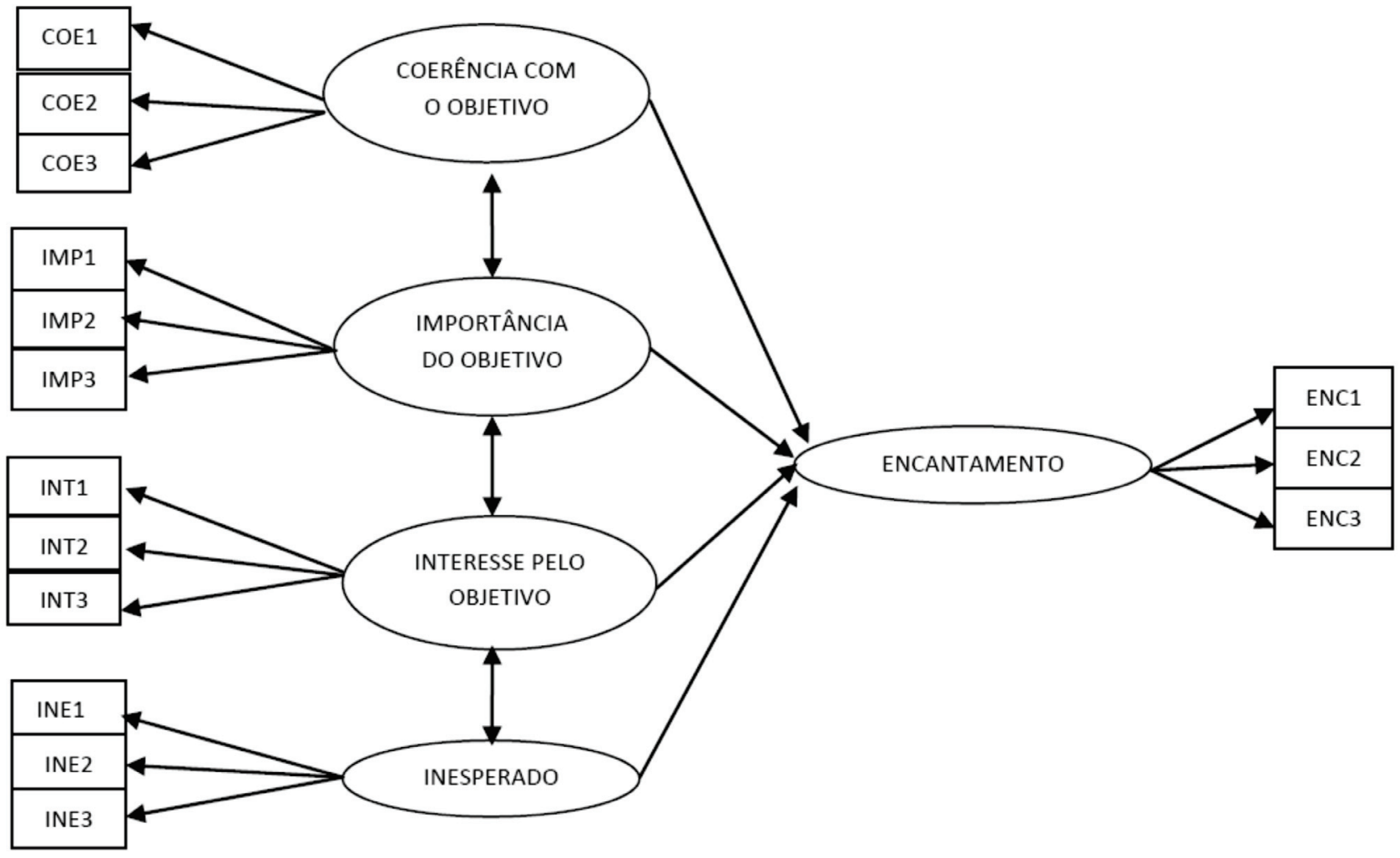

Fonte: Adaptado de Ma et al. (2013, p. 372).

Os dados obtidos a partir dos questionários tiveram seu processamento realizado através do Statistical Package for the Social Sciences [SPSS], versão 19.0 para Windows, utilizando como procedimento de análise, a Análise Fatorial Exploratória [AFE] e a Análise de Regressão Linear Múltipla.

Os resultados encontrados são apresentados e discutidos a seguir.

RESULTADOS E DISCUSSÕES

\section{ANÁLISE DO MODELO DE MENSURAÇÃO}

Para se verificar a adequação das variáveis observáveis ao modelo de relacionamento adotado, foi proposta a Análise Fatorial Exploratória [AFE]. A AFE é uma técnica de análise exploratória de dados que tem por objetivo descobrir e analisar a estrutura de um conjunto de variáveis inter-relacionadas de modo a construir uma escala de medida para fatores (intrínsecos) que de alguma forma (mais ou menos explícita) controlam as variáveis originais (Marôco, 2010). Esta 
ISSN: 1983-7151

técnica usa as correlações observadas entre as variáveis originais para estimar o(s) fator(es) comum(ns) e as relações estruturais que ligam os fatores (latentes) às variáveis.

Visando a conformidade com os pressupostos da Análise Fatorial Exploratória (AFE), foi realizada uma inspeção na matriz de correlações, em que são consideradas para cada variável, as correlações, índices de antiimagem, comunalidade e cargas fatoriais. Os procedimentos de adequação aos pressupostos da AFE são os seguintes (Hair, Júnior. Black, Babin, Anderson, \& Tatham, 2009):

- Anti-imagem: corresponde à matriz das correlações parciais entre variáveis após a Análise Fatorial, representa o grau que os fatores explicam um ao outro resultado. Os valores de referência devem ser igual ou maior que 0,5.

- Comunalidade: corresponde à quantia total de variância que uma variável original compartilha com todas as outras variáveis incluídas na análise. Os valores de referência devem ser igual ou maior que 0,6.

- Carga Fatorial: corresponde à correlação entre as variáveis originais e os fatores, bem como a chave para o entendimento da natureza de um fator em particular. Os valores de referência devem ser igual ou maior que 0,5 em uma dimensão.

Para a realização da AFE, foi utilizado, como método de extração, a Análise dos Componentes Principais [ACP], já que procurou-se levar em consideração a variância total dos dados. Como método de rotação de fatores, foi utilizado o Varimax, visando minimizar a ocorrência de uma variável possuir alta carga fatorial em mais de uma dimensão. Também foi utilizado, como critério para a constituição dos fatores, o autovalor (Eigenvalue), ou seja, o quanto o fator consegue explicar a variância total dos dados. No caso, apenas foram considerados constructos (fatores) com autovalores superiores a 1 (um).

A análise da matriz de correção é apresentada na tabela 01, considerandose a retirada dos outliers:

Tabela 01 - Índices calculados da análise da matriz de correlação sem outliers 
Dor: 10.14210/rtva.v18n2.p103-127

\begin{tabular}{|c|c|c|c|c|}
\hline Variável & Anti-imagem & Comunalidade & Cargas Fatoriais & $\begin{array}{c}\text { Alfa de } \\
\text { Cronbach* }\end{array}$ \\
\hline \multicolumn{2}{|c|}{ Coerência com o Objetivo } & & & 0,653 \\
\hline COE1 & 0,704 & 0,512 & 0,713 & \\
\hline COE2 & 0,628 & 0,626 & 0,842 & \\
\hline COE3 & 0,622 & 0,640 & 0,749 & \\
\hline \multicolumn{3}{|c|}{ Importância do Objetivo } & & 0,638 \\
\hline IMP1 & 0,664 & 0,570 & 0,755 & \\
\hline IMP2 & 0,657 & 0,581 & 0,762 & \\
\hline IMP3 & 0,642 & 0,608 & 0,780 & \\
\hline \multicolumn{3}{|c|}{ Interesse pelo Objetivo } & & 0,589 \\
\hline INT1 & 0,633 & 0,563 & 0,750 & \\
\hline INT2 & 0,658 & 0,521 & 0,721 & \\
\hline INT3 & 0,623 & 0,585 & 0,765 & \\
\hline Inesperado & & & & 0,662 \\
\hline INE1 & 0,628 & 0,658 & 0,811 & \\
\hline INE2 & 0,685 & 0,562 & 0,750 & \\
\hline INE3 & 0,661 & 0,595 & 0,771 & \\
\hline Encantamentc & & & & 0,542 \\
\hline ENC1 & 0,540 & 0,672 & 0,820 & \\
\hline ENC2 & 0,543 & 0,635 & 0,797 & \\
\hline ENC3 & 0,705 & 0,273 & 0,523 & \\
\hline
\end{tabular}

Fonte: Dados da pesquisa (2014).

* Alfa de Cronbach estimado após a exclusão das variáveis que não se adequaram aos critérios propostos quando da inspeção da matriz de correlação.

Observa-se que todos os constructos apresentam um valor de Alfa de Cronbach próximo ao valor mínimo estabelecido como critério de aceitação, ou seja, valor de Alfa de 0,6 em estudos de Ciências Sociais Aplicadas, (Hair Jr. et al., 2009). Os índices de Anti-imagem, Comunalidade e Cargas Fatoriais apresentam valores próximos aos critérios estabelecidos para análise, nas variáveis observáveis de todos os construtos, com exceção da variável ENC3 (Eu não teria palavras para descrever como foi boa a visita ao Magic Kingdom) no que se refere ao índice "Comunalidade" que, na avaliação de pressupostos, mostrou-se abaixo do critério utilizado, já que neste índice, os valores de referência devem ser iguais ou maiores que 0,6. Destaca-se que se optou por utilizar aquelas variáveis que apresentaram comunalidades abaixo de 0,6, mas muito próximas deste valor. 
Na tabela 02 são apresentadas as variáveis excluídas por meio da análise da matriz de correlação e os critérios de exclusão.

Tabela 02 - Variáveis excluídas por meio da análise da matriz de correlação - critérios de exclusão

Variável

Critério de exclusão
Índice Padrão

Alfa de Cronbach da dimensão (recalculado)

Eu não teria palavras par dizer como foi boa a visita ao Magic Kingdom

ENC3

Fonte: Dados da pesquisa (2014).

Como no modelo resultante de relacionamento de variáveis, somente se verifica a presença de uma única equação de regressão, entendeu-se mais viável utilizar-se a análise de regressão linear múltipla para estudar os relacionamentos existentes entre as dimensões do modelo. Desta forma, não foi necessário a realização da Análise Fatorial Confirmatória (AFC).

As cargas fatoriais do modelo de mensuração obtido e utilizadas para a realização da análise de regressão linear múltipla são apresentadas na tabela 03:

Tabela 03 - Cargas fatoriais do modelo de medida

\begin{tabular}{|c|c|c|c|c|c|}
\hline Variável & $\begin{array}{c}\text { Coerência com o } \\
\text { Objetivo }\end{array}$ & $\begin{array}{c}\text { Importância do } \\
\text { Objetivo }\end{array}$ & $\begin{array}{c}\text { Interesse pelo } \\
\text { Objetivo }\end{array}$ & Inesperado & Encantamento \\
\hline COE1 & 0,713 & & & & \\
\hline COE2 & 0,842 & & & & \\
\hline COE3 & 0,749 & & & & \\
\hline IMP1 & & 0,755 & & & \\
\hline IMP2 & & 0,762 & & & \\
\hline IMP3 & & 0,780 & & & \\
\hline INT1 & & & 0,750 & & \\
\hline INT2 & & & 0,721 & & \\
\hline INT3 & & & 0,765 & & \\
\hline INE1 & & & & 0,811 & \\
\hline INE2 & & & & 0,750 & \\
\hline INE3 & & & & 0,771 & \\
\hline ENC1 & & & & & 0,842 \\
\hline ENC2 & & & & & 0,842 \\
\hline
\end{tabular}

Fonte: Dados da pesquisa (2014). 
As variáveis resultantes deste processo foram consideradas adequadas para a etapa de análise de regressão linear múltipla do modelo.

\section{ANÁLISE DE REGRESSÃO LINEAR MÚLTIPLA DO MODELO}

O objetivo desta análise foi analisar e prever o comportamento das variáveis consideradas como antecedentes do Encantamento (Coerência com o Objetivo, Importância do Objetivo, Interesse pelo Objetivo e Inesperado), visando criar subsídios para uma melhor tomada de decisão. Em outras palavras, o intuito foi verificar se uma ou mais variáveis dependentes estão relacionadas com a variável dependente "Encantamento" e qual a natureza dessa relação.

Para investigar o grau de correlação entre as variáveis em estudo foi utilizado o coeficiente de correlação de Pearson. Duas variáveis são consideradas altamente correlacionadas se as mudanças ocorridas em uma delas estiverem fortemente associadas com as mudanças ocorridas na outra (Corrar, Paulo, \& Dias, 2009). O indicador $\mathrm{R}^{2}$ pode ser obtido tomando-se o valor do coeficiente de correlação $r$ e elevando-se ao quadrado $\left(R^{2}\right)$. $O R^{2}$ é o coeficiente de determinação ou poder explicativo da regressão. Indica quanto da variação na variável dependente é explicado pelas variações nas variáveis independentes.

Também foi utilizado o teste ANOVA [Análise de Variância], que tem por finalidade testar o efeito do conjunto de variáveis independentes sobre a variável dependente. Consiste em verificar a probabilidade de que os parâmetros da regressão em conjunto sejam iguais a zero. Em outras palavras, significa verificar se a combinação linear das variáveis independentes exerce influência significativa ou não sobre a variável dependente.

Na tabela 04 é apresentada a matriz de correlação das variáveis latentes obtidas no estudo:

Tabela 04 - Matriz de correlação das variáveis latentes obtidas no estudo

\begin{tabular}{c|c|c|c|c|c|c}
\hline \multicolumn{2}{|c|}{ Dimensões } & A & B & C & D & E \\
\hline \multirow{2}{*}{ A } & Coef. de Correlação de Pearson & 1 & \multicolumn{4}{|l}{} \\
& Sig. (2 extremidades) & - & & \\
\multirow{2}{*}{ B } & Coef. de Correlação de Pearson & 0,139 & 1 & & \\
& Sig. (2 extremidades) & 0,070 & - & & \\
\hline
\end{tabular}




\begin{tabular}{c|c|c|c|c|c|c}
\hline \multirow{2}{*}{ C } & Coef. de Correlação de Pearson & 0,092 & $0,708^{* *}$ & 1 & \multicolumn{2}{|c}{} \\
& Sig. (2 extremidades) & 0,233 & 0,000 & - & \multicolumn{2}{|c}{} \\
\hline \multirow{2}{*}{$\mathrm{D}$} & Coef. de Correlação de Pearson & $0,439^{* *}$ & $0,560^{* *}$ & $0,471^{* *}$ & 1 & \\
& Sig. (2 extremidades) & 0,000 & 0,000 & 0,000 & - & \\
\hline \multirow{2}{*}{$\mathrm{E}$} & Coef. de Correlação de Pearson & $0,372^{* *}$ & $0,214^{* *}$ & $0,308^{* *}$ & $0,381^{* *}$ & 1 \\
& Sig. (2 extremidades) & 0,000 & 0,005 & 0,000 & 0,000 & - \\
\hline
\end{tabular}

Fonte: Dados da pesquisa (2014).

*. A correlação é significativa ao nível de 0,05

**. A correlação é significativa ao nível de 0,01

Legenda:

A: Coerência com o Objetivo, B: Importância do Objetivo, C: Interesse pelo Objetivo, D: Inesperado, E: Encantamento

Sig. (2 extremidades): corresponde ao valor de probabilidade de erro (p) em se aceitar a hipótese alternativa do estudo ( $\mathrm{Ha}$ ). No caso, Ha é entendida como a existência de correlação entre as variáveis analisadas.

Conforme pode ser observado através dos dados apresentados na tabela 4, em função dos valores obtidos dos coeficientes de Pearson ( $r$ ) e o nível de significância da relação (p), existe correlação moderada, significativa e positiva entre todas as dimensões consideradas como antecedentes do Encantamento (Coerência com o Objetivo, Importância do Objetivo, Interesse pelo Objetivo e Inesperado) e a variável dependente "Encantamento". No caso, considera-se como relação significativa quando o valor de $p$ for inferior a 0,05. Considerase que coeficientes de Pearson entre 0,3 e 0,6 apresenta correlação linear moderada de variáveis.

É interessante verificar a forte correlação existente entre "Importância do Objetivo" e "Interesse do Objetivo", cujo valor do coeficiente de correlação de Pearson foi de 0,708 , significativo ao nível de $1 \%$ de probabilidade de erro. Este resultado era esperado na medida que o "Interesse pelo objetivo" pode decorrer das experiências com situações percebidas como importantes, o que pode provocar uma emoção positiva.

Na tabela 5 é apresentada a análise de variância [NOVA], obtida quando da ocorrência do teste de regressão linear múltipla. 
Tabela 05 - Análise de Variância (ANOVA)

\begin{tabular}{c|c|c|c|c|c|c}
\hline \multicolumn{2}{c|}{ Modelo } & $\begin{array}{c}\text { Soma dos } \\
\text { Quadrados }\end{array}$ & $\begin{array}{c}\text { Graus de } \\
\text { Liberdade }\end{array}$ & Quadrado Médio & Z & Sig. \\
\hline \multirow{3}{*}{1} & Regressão & 40,451 & 4 & 10,113 & 12,958 & $<0,0001$ \\
\cline { 2 - 7 } & Resíduo & 129,549 & 166 & 0,780 & & \\
\cline { 2 - 8 } & Total & 170,000 & 170 & & & \\
\hline
\end{tabular}

Fonte: Dados da pesquisa (2014).

Pelos dados apresentados, observa-se que existem correlações significativas entre as variáveis independentes utilizadas no estudo (Coerência com o Objetivo, Importância do Objetivo, Interesse pelo Objetivo e Inesperado) e a variável dependente "Encantamento", visto que o nível de significância ( $p$ ) foi inferior à 0,0001 . Desta forma, é possível estimar-se agora os coeficientes de regressão a partir da análise de regressão múltipla, cujos resultados são apresentados na tabela 6:

Tabela 6 - Coeficientes de Regressão

\begin{tabular}{|c|c|c|c|c|c|c|}
\hline & \multirow{2}{*}{ Modelo } & \multicolumn{2}{|c|}{ Coeficientes não padronizados } & $\begin{array}{l}\text { Coeficientes } \\
\text { padronizados }\end{array}$ & \multirow[t]{2}{*}{$\mathrm{T}$} & \multirow{2}{*}{ Sig. } \\
\hline & & B & Erro Padrão & Beta & & \\
\hline \multirow{5}{*}{1} & (Constante) & $3,381 \mathrm{E}-16$ & 0,068 & & 0,000 & 1,000 \\
\hline & COERENCIA & 0,276 & 0,076 & 0,276 & 3,607 & 0,000 \\
\hline & IMPORTANCIA & $-0,141$ & 0,103 & $-0,141$ & $-1,364$ & 0,174 \\
\hline & INTERESSE & 0,286 & 0,097 & 0,286 & 2,956 & 0,004 \\
\hline & INESPERADO & 0,204 & 0,092 & 0,204 & 2,212 & 0,028 \\
\hline
\end{tabular}

Fonte: Dados da pesquisa (2014).

O método de estimativa utilizado foi o Método dos Mínimos Quadrados (MMQ) que tem o objetivo de obter a menor soma de quadrados dos resíduos possível. O método de busca sequencial das variáveis, utilizado neste procedimento, foi o da inclusão generalizada de todas as variáveis.

Observa-se que, pelo procedimento estatístico utilizado, no modelo final de regressão, somente a variável independente "Importância do Objetivo" não apresentou relação significativa com a variável dependente "Encantamento" (valor de probabilidade de erro de 0,174$)$. Tal fato pode ser consequência da existência de multicolineariedade entre as variáveis utilizadas no modelo, ou seja, quando duas variáveis independentes forem altamente correlacionadas entre si, 
ISSN: 1983-7151

o que acontece entre "Importância do Objetivo" e "Interesse do Objetivo", e ainda forem correlacionadas com a variável dependente, que é o caso, é provável que apenas uma delas entre no modelo, haja visto que quando a primeira delas for incluída, sobrará pouco poder explicativo adicional para a outra.

Observa-se que o relacionamento das demais variáveis independentes é homogêneo, com coeficiente padronizado Beta na ordem de 0,2. Ou seja, as variáveis dependentes "Coerência com o Objetivo", "Interesse pelo Objetivo" e "Inesperado" apresentam comportamento semelhante em influenciar a variável dependente "Encantamento".

A Tabela 07 apresenta o Coeficiente de determinação (R2) obtido.

Tabela 7 - Coeficiente de Determinação (R2)

\begin{tabular}{c|c|c|c|c}
\hline Modelo & $\mathrm{R}$ & R quadrado & R quadrado ajustado & Erro padrão da estimativa \\
\hline 1 & 0,488 & 0,238 & 0,220 & 0,88341274 \\
\hline
\end{tabular}

Fonte: Dados da pesquisa (2014).

Pelos dados apresentados, apesar de significativo, observa-se que, no conjunto, a variância do conjunto de variáveis independentes "Coerência com o Objetivo", "Interesse pelo Objetivo" e "Inesperado" têm um poder explicativo fraco sobre a variância da variável dependente "Encantamento". Isto indica que, apesar da existência de relacionamento moderado, em sua forma individual, de cada variável independente com a variável dependente "Encantamento", expresso através dos valores apresentados na Matriz de Correlação (Tabela 04), no conjunto do modelo, tal relacionamento é fraco (R2 inferior à 0,3 ). Nesta situação, presume-se que há outras variáveis capazes de influenciar o "Encantamento" do cliente além daquelas utilizadas neste modelo de análise.

Os resultados obtidos nesta investigação podem ser correlacionados com estudos anteriores sobre a Encantamento e emoções relacionadas, como por exemplo, Ma et al. (2013), no qual foi verificado que as dimensões "Coerência com o Objetivo", "Interesse pelo Objetivo" e "Inesperado" têm um impacto significativo no "Encantamento". Tal fato foi observado empiricamente na pesquisa de campo, no momento da aplicação do instrumento de pesquisa. Os adolescentes que se apresentavam mais encantados mostravam que a viagem 
estava coerente com seus objetivos, possuíam interesse na visitação ao parque e encontraram o fator "inesperado" no parque.

Estudos de Ellsworth e Smith (1988 a,b), constataram a existência de relação entre coerência com o objetivo e a novidade (dimensão na qual se encontra o inesperado) e que estas são dimensões utilizadas para conceituar a emoção de Encantamento. Resultados encontrados nos estudos realizados por Roseman e Smith (2001) e Scherer (1997) apontam que o Inesperado é um conceito similar ao de surpresa e estímulos de surpresa fazem parte da formação da emoção de Encantamento. Scherer (1993) demonstrou que algumas dimensões, entre elas a coerência com o objetivo podem provocar a emoção de Encantamento.

\section{CONCLUSÕES E RECOMENDAÇÕES}

O presente estudo teve como objetivo investigar as inter-relações entre alguns fatores capazes de influenciar o Encantamento do Cliente. Entende-se que os resultados obtidos contribuem para os estudos relativos à hipótese de que existe uma relação significativa entre "Coerência do Objetivo", "importância do Objetivo" "Interesse pelo Objetivo" e "Inesperado" e "Encantamento", ou seja, estas dimensões apresentam impactos significativos na formação do Encantamento do Cliente.

Estes resultados podem ser comparados com os encontrados por Ma et al. (2013), Ellsworth e Smith (1988 a,b), Roseman e Smith (2001) e Scherer (1993,1997). Além disso, tal fato foi observado empiricamente na pesquisa de campo, uma vez que os adolescentes que estavam mais encantados mostravam que a viagem estava coerente com seus objetivos, possuíam interesse pela visitação ao parque e que também durante a visitação encontraram o fator inesperado.

A partir da análise dos relacionamentos do modelo foi obtido o valor do coeficiente de determinação $\left(R^{2}\right)$ de 0,238 , o que significa dizer que, para a população estudada, o modelo de relacionamento proposto apresentou uma relação fraca. De uma forma geral, observou-se que a dimensão "Encantamento", neste estudo, apresenta-se muito mais ligada ao valor da experiência (Afetiva) do que às informações prévias (Cognitiva). 
Os resultados encontrados neste estudo trazem implicações gerenciais tanto para o próprio parque temático como para a agência responsável pela excursão. Neste sentido, entende-se que tanto agências que realizam excursões para os parques temáticos, como o próprio parque temático podem enfatizar o uso da afetividade no processo de venda dos produtos e serviços, desenvolvendo ações focadas na experiência vivida por estes adolescentes durante todo o processo da viagem e da visita ao parque.

Deve-se destacar que os questionários foram aplicados com adolescentes, ficando, assim, difícil generalizar resultados para outros públicos. Para melhorar a generalização dos resultados a partir deste estudo é recomendada a reaplicação deste procedimento de pesquisa com diferentes faixas etárias. Além disso, futuras investigações poderiam contemplar um numero maior de constructos.

Observou-se também dificuldade na aplicação do questionário enquanto instrumento de pesquisa, principalmente em relação à falta de concentração dos adolescentes e a dificuldade para tratar sobre emoções com pesquisa quantitativa. Desta forma, sugere-se um aprimoramento do instrumento de pesquisa para a coleta das informações em pesquisas futuras sobre o tema.

\section{REFERÊNCIAS}

Almeida, S. O. (2003) Avaliação pós-consumo: proposição de uma escala para mensuração do encantamento do cliente. Dissertação de mestrado, Universidade Federal do Rio Grande do Sul, Porto Alegre, Brasil.

Almeida, S. O., Nique, W. M. (2007) Encantamento do cliente: proposiçăo de uma escala para mensuraçăo do constructo. Revista de Administração Contemporânea, 11 (4), 109-130.

Ansarah, M. G. R. (1999) Turismo, segmentação de mercado. São Paulo: Futura.

Arnold, M.J. \& Reynoldsb, K.E. (2005) Customer delight in a retail context: investigating delightful and terrible shopping experiences. Journal of Business Research, 1132- 1145.

Arriffin, A.A.M \& Yahaya, M.F. (2013) The relationship between airport image, national identity and passengers delight: a case study of the Malaysian low cost carrier terminal (LCCT). Journal of Air Transport Management, p. 33- 36.

Barretto, M. (1991) Planejamento e organização em turismo (9a ed.) Campinas: Papirus. 
Bee, C. \& Neubaum, D. O. (2014) The role of cognitive appraisal and emotions of family members in the family business system. Journal of Family Business Strategy, 323-333.

Beni, M. C. (2014) Política e planejamento do turismo no Brasil. In: Semana do Turismo da UFRN, 10, 2014. Palestra. Natal, Rio Grande do Norte, Brasil.

Berman, B. (2005) How to delight your customers. Califórnia Management Review, 48, p.129-151.

Bollen, K.A. (1989) Structural equations wint latent variables. New York: Wiley.

Chagas, M. M. (2010) Análise da relação causal entre imagem de destinos, qualidade, satisfação e fidelidade: um estudo de acordo com a percepção do turista nacional no destino turístico Natal. Dissertação de mestrado, Universidade Federal do Rio Grande do Norte, Natal, Brasil.

Conellan. T. (2010) Nos bastidores da Disney: os segredos do sucesso da mais poderosa empresa de diversões do mundo. São Paulo: Saraiva.

Corrar, L. J., Paulo, E., \& Dias Filho, J. M. (2009) Análise multivariada: para os cursos de administração, ciências contábeis e economia. São Paulo: Atlas.

Crotts, J.C. \& Magnini, V.P. (2011) The customer delight construct: Is Surprise Essential? Annals of Tourism Research, 38 (2), 719-722.

Ellsworth, P. C. \& Smith, C. A. (1998a) Shades of joy: Patterns of appraisal differentiating pleasant emotions. Cognition and emotion, 2(4), 301-331.

Ellsworth, P. C. (1998b) From appraisal to emotion: Differences among unpleasant feelings. Motivation and Emotion, 12(3), 271-302.

Gohr, C. F. \& Santos, L. C. (2010) Estratégias Competitivas para Empresas Turísticas: um estudo no setor hoteleiro de um município do litoral catarinense. Turismo em análise, 21 (2), 297-319.

Hair Júnior, F., Black, W. C., Babin, B. J., Anderson, R. E., \& Tatham, R. L. (2009). Análise multivariada de dados. Bookman Editora.

Hosany, S. (2012) Appraisal determinants of tourist emotional responses. Journal of Travel Research, 51(3), 303-314.

Johnson, M. D., Anderson, E. W., \& Fornell, C. (1995) Rational and adaptive performance expectations in a customer satisfaction framework. Journal of Consumer Research, 21, 695-707.

Johnson, A. R. \& Stewart, D.W. (2005) A reappraisal of the role of emotion in consumer behavior: Traditional and contemporary approaches. Review of Marketing Research, 3-33. 
Füller, J. \& Matzler, K. (2008) Customer delight and market segmentation: An application of the three-factor theory of customer satisfaction on life style groups. Tourism Management, 116 -126.

Gastal. S. \& Castrogiovanni, C. (2003) Turismo na pós-modernidade (des) inquietações. Porto Alegre: EDIPUCRS.

Gentina, E., Butori, R., Rose, G.M., \& Bakir, A. (2014) How national culture impacts teenage shopping behavior: Comparing French and American consumers. Journal of Business Research, 464-470.

Keiningham, T. \& Vavra, T. (2001) The customer delight principe. New York: McGraw-HillAmerican Marketing Association.

Kotler, P., Bowen, J., \& Makens, J. (1999) Marketing for hospitality and Tourism (2a ed.) New Jersey: Prentice-Hall International.

Kumar, A. (1996) Customer delight: creating and maintaining competitive advantage. Thesis of doctor, Indiana University, Indiana, EUA.

Kwong, K.K. \& Yau, O.H.M. (2002) The conceptualization of customer delight: a research framework. Asia Pacific Management Review, 255-266.

Lerner, J.S. \& Keltner, D. (2000) Beyond valence: toward a model of emotion-specific influences on judgment and choice. Cognition and Emotion, 473-493.

Lohmann, G. \& Panosso Netto, A. (2008) Teoria do turismo: conceitos, modelos e sistemas. São Paulo: Aleph.

Ma, J. (2013) Emotions derived from theme park experiences: the antecedents and consequences of customer delight. Thesis of doctor, University of Queensland, Austrália.

Ma, J., Gao, J., Scott, N., \& Ding, P. (2013). Customer delight from theme park experiences: The antecedents of delight based on cognitive appraisal theory. Annals of Tourism Research, 42, 359-381.

Manosso, F. C., Gândara, J. M. G., Souza, T., \& Bógea, V. (2012). A gestão das emoções dos hóspedes. Revista brasileira de pesquisa em turismo, 6(3), 357-374.

Marôco, J. (2010) Análise de equações estruturais: Fundamentos teóricos, software e aplicações. Perô Pinheiro: Report Number.

Masroujeh, R. (2009) Critical factors for customer satisfaction and delight in the palestinian pharmaceutical market. Dissertation of Master, Islamic University, Gaza.

Organização Mundial do Turismo [OMT]. Commited to tourism, travel and the millennium development goals. Recuperado em 11 outubro, 2016, de http://www2.unwto.org/. 
Ramos, B. A \& Dias, R. (2010) Aspectos de competitividade e complementaridade nos circuitos turísticos de minas gerais. Caderno Virtual de Turismo, 10 (3), 15-24.

Robinson, G.J. (2012) Delight, satisfaction and behavioral intentions in a hospital setting: the role of environmental and interpersonal services. Thesis of doctor, Cleveland State University, Cleveland, 2012.

Roseman, I. J. \& Smith, C. A. (2001) Appraisal theory: Overview, assumptions, varieties, controversies. In T. Johnstone (Ed.), Appraisal Processes in Emotion: Theory, Methods, Research. New York: Oxford University Press.

Santil, J. A. S. (2001) Os parques temáticos no contexto da atividade turística. Turismo Visão e Ação, 7, 83-93.

Santoro, M. A. G. (2014) Análise da relação entre apego ao lugar, satisfação e fidelidade dos visitantes em destinos turísticos ambientais: um estudo em Fernando de Noronha/PE. Dissertação de mestrado, Universidade Federal do Rio Grande do Norte, Natal, Brasil.

Scherer, K. R. (1993) Studying the emotion-antecedent appraisal process: An expert system approach. Cognition \& Emotion, 7 (3-4), 325-355.

Scherer, K. R. (1997) Profiles of emotion-antecedent appraisal: Testing theoretical predictions across cultures. Cognition \& Emotion, 11(2), 113-150.

Schümmer, B. (2007) Cognitive and affective antecedents of and behavioral intentions connected to delight, satisfaction, dissatisfaction, and outrage in the dutch academic education market. Thesis of International Business, Faculty of Economics and Business Administration, University Maastricht, Maastricht.

Silva, F. B. (2004) A psicologia dos serviços em turismo e hotelaria: entender o cliente e atender com eficiência. São Paulo: Pioneira Thomson Learning.

Tacconi, M.F. F.S. (2012) A confiança interorganizacional nas compras. Tese de doutorado, Universidade Federal do Rio Grande do Norte, Natal, Brasil.

TEA/AECOM. Global attractions attendance report. (2016) Recuperado em 18 outubro, 2016, de http://www.teaconnect.org/images/files/TEA_160_611852_160525.pdf.

Torres, E.N., Fu, X. \& Lehto, X. (2014) Examining key drivers of customer delight in a hotel experience: A cross-cultural perspective. International Journal of Hospitality Management, 255- 262.

Valente, S. B. (2006) Análise de visitação dos parques de diversões brasileiros como opção de lazer. Dissertação de mestrado, Universidade de São Paulo, São Paulo, Brasil. 
World Travel \& Tourism Council. World travel \& tourism world economic impact 2016. Recuperado em 11 outubro, 2016, de http://www.wttc.org/.

Contribuição dos autores na construção do trabalho:

Silva: Fundamentação teórica, elaboração da metodologia, elaboração do instrumento de pesquisa, aplicação do instrumento de pesquisa, discussão dos resultados, conclusões e recomendações.

Marques Júnior: Elaboração da Metodologia, elaboração do instrumento de pesquisa, análise dos resultados, discussões do resultado, análise do modelo de mensuração, conclusões e recomendações. 